\title{
WIND-INTERACTION MODELS FOR THE EARLY AFTERGLOWS OF GAMMA-RAY BURSTS: THE CASE OF GRB 021004
}

\author{
Zhi-Yun Li and Roger A. Chevalier \\ Department of Astronomy, University of Virginia, P.O. Box 3818 \\ Charlottesville, VA 22903; zl4h@virginia.edu, rac5x@virginia.edu
}

Received __; accepted _ 


\begin{abstract}
Wind-interaction models for gamma-ray burst afterglows predict that the optical emission from the reverse shock drops below that from the forward shock within 100s of seconds of the burst. The typical frequency $\nu_{m}$ of the synchrotron emission from the forward shock passes through the optical band typically on a timescale of minutes to hours. Before the passage of $\nu_{m}$, the optical flux evolves as $t^{-1 / 4}$ and after the passage, the decay steepens to $t^{-(3 p-2) / 4}$, where $p$ is the exponent for the assumed power-law energy distribution of nonthermal electrons and is typically $\sim 2$. The steepening in the slope of temporal decay should be readily identifiable in the early afterglow light curves. We propose that such a steepening was observed in the R-band light curve of GRB 021004 around day 0.1. Available data at several radio frequencies are consistent with this interpretation, as are the X-ray observations around day 1. The early evolution of GRB 021004 contrasts with that of GRB 990123, which can be described by emission from interaction with a constant density medium.
\end{abstract}

Subject headings: gamma-rays: bursts — stars: mass loss — stars: supernovae: general 


\section{INTRODUCTION}

The initial model for the afterglows of gamma-ray bursts (GRBs) involved synchrotron emission from nonthermal electrons accelerated to a power-law spectrum in a relativistic spherical blast wave expanding into a constant-density, presumably interstellar, medium (ISM; Mészáros \& Rees 1997). This simplest model has difficulty, however, explaining quantitatively the dozen or so sources whose afterglows are observed well enough to allow for detailed modeling (e.g., Panaitescu \& Kumar 2002). The most commonly discussed complication is a collimated energy injection (Rhoads 1997; Sari, Piran \& Halpern 1999). This jet model provides a reasonable fit to the majority of the well observed afterglows, assuming a constant-density ambient medium (Panaitescu \& Kumar 2002). For some sources, a blast wave expanding into an ambient medium of $r^{-2}$ density distribution, as expected of a stellar wind, can fit the data equally well or even better (Chevalier \& Li 2000, CL00 hereafter; Li \& Chevalier 2001; Panaitescu \& Kumar 2002). Notable examples include GRB 970508 (see Frail, Waxman \& Kulkarni 2000 for a different view) and GRB 011121 (Price et al. 2002). There is evidence for two types of GRB ambient environments, with implications for their progenitors. They are not immediately distinguishable because, at an age of a few days, the preshock wind density is comparable to an interstellar density. At earlier times, the density contrast is higher and the jet effects are less important. The early afterglow observations are expected to provide a better handle on the nature of the ambient medium.

In this Letter, we summarize the characteristics of the early afterglows expected in the wind model $(\S 2)$, and argue that the afterglow observations of GRB 021004 are consistent with the source interacting with a Wolf-Rayet type wind $(\S 3)$. The strongest evidence for wind interaction comes from the initial slow decay of the R-band light curve and its prominent steepening around day 0.1 . 


\section{ANALYTIC LIGHT CURVES OF EARLY AFTERGLOWS}

Analytic light curves for the standard ISM model are given in Sari, Piran \& Narayan (1998), assuming a power-law electron energy spectrum with index $p$ and constant fractions of blast wave energy, $\epsilon_{e}$ and $\epsilon_{B}$, going into nonthermal electrons and magnetic field respectively. At any given time, the synchrotron spectrum is determined by a set of characteristic frequencies: the typical frequency $\nu_{m}$, cooling frequency $\nu_{c}$, and self-absorption frequency $\nu_{a}$. The light curve at any given frequency $\nu$ is determined by the characteristic times $t_{m}, t_{c}$ and $t_{a}$, when $\nu_{m}, \nu_{c}$ and $\nu_{a}$ cross $\nu$, and the critical time $t_{0}$ when $\nu_{m}$ and $\nu_{c}$ become equal. The light curve of the ISM model was extended to the wind case by CL00 (see also Panaitescu \& Kumar 2000 and Granot \& Sari 2002). In the wind model, the cooling and self-absorption frequencies are expected to be lower at early times than those in the ISM model, because of a higher ambient density at small radii. The cooling frequency has a strong effect on the early emission in the optical and the self-absorption frequency in radio.

At the earliest times, the optical flux may be dominated by the emission from the reverse shock front, but the forward shock front is not much fainter for wind interaction (CL00, eq. [58]), assuming that the two shocks have similar efficiencies for the production of synchrotron radiation. Once the reverse shock front has passed through the initial shell, which is expected to occur on a timescale similar to that of the gamma-ray burst, the reverse shock emission drops sharply because it is in the fast cooling regime. Unless the reverse shock is "refreshed" by a continued flow, the decline is determined by off-axis

emission that arrives at a later time (Kumar \& Panaitescu 2000). Optical frequencies are expected to be between $\nu_{c}$ and $\nu_{m}$, so the flux is $\sim \nu^{-1 / 2}$ and decays as $t^{-5 / 2}$ (Kumar \& Panaitescu 2000).

To describe the forward shock emission, we use the characteristic times mentioned 
above, which were estimated by CL00 assuming $p=2.5$. We rescale the estimates to the R-band with $\nu_{R}=\left(\nu / 4.5 \times 10^{14} \mathrm{~Hz}\right)$, and obtain

$$
t_{m}=0.04(1+z)^{1 / 3} \epsilon_{e,-1}^{4 / 3} \epsilon_{B,-1}^{1 / 3} E_{52}^{1 / 3} \nu_{R}^{-2 / 3} \text { days, }
$$

which is about an hour for standard parameters. The parameter $z$ is the cosmological redshift, $E_{52}$ is the blast wave energy in units of $10^{52} \mathrm{ergs}$, and $\epsilon_{n}=\epsilon / 10^{n}$. Note that $t_{m}$ does not depend on the wind density $A$ directly, where $\rho=A r^{-2}$, although the wind must be sufficiently dense that the transition from fast cooling to slow cooling occurs after $t_{m}$. It depends most sensitively on $\epsilon_{e}$. The condition on $\epsilon_{e}$ for the R-band break to occur between one minute and one hour is $0.005<\epsilon_{e}(1+z)^{1 / 4} \epsilon_{B,-1}^{1 / 4} E_{52}^{1 / 4}<0.1$, which covers a reasonable range, and has only a weak dependence on other parameters. The transition to slow cooling occurs around the time $t_{0}=1(1+z) \epsilon_{e,-1} \epsilon_{B,-1} A_{*}$ day, which is typically later than $t_{m}$ in the R-band. Here, $A_{*}=A / 5 \times 10^{11} \mathrm{~g} \mathrm{~cm}^{-1}$. The cooling frequency crosses the frequency $\nu_{R}$ at a time $t_{c}=5 \times 10^{3}(1+z)^{3} \epsilon_{B,-1}^{3} E_{52}^{-1} A_{*}^{4} \nu_{R}^{2}$ days, which is typically later than both $t_{m}$ and $t_{0}$. The self-absorption frequency $\nu_{a}$ is typically well below the optical, and can be ignored.

Before $t_{0}$, the synchrotron electrons are in the fast cooling regime, and the flux peaks at the cooling frequency $\nu_{c}$, so $F_{\nu_{c}}=F_{\nu, \max }$ with

$$
F_{\nu, \max }=2.1(1+z)^{3 / 2} \epsilon_{B,-1}^{1 / 2} E_{52}^{1 / 2} A_{*} d_{L 1}^{-2} t_{\text {days }}^{-1 / 2} \text { mJy }
$$

where $d_{L 1}$ is the luminosity distance in units of $10 \mathrm{Gpc}$. The flux at the typical frequency $\nu_{m}$ is lower, and is given by

$$
F_{\nu_{m}}=2.7(1+z)^{1 / 2} \epsilon_{e,-1}^{-1} \epsilon_{B,-1}^{-1 / 2} E_{52}^{1 / 2} d_{L 1}^{-2} t_{\text {days }}^{1 / 2} \quad \mathrm{mJy}
$$

which is independent of the wind density. After the transition to slow cooling at $t_{0}$, the flux peaks at $\nu_{m}$ rather than $\nu_{c}$, and now $F_{\nu_{m}}=F_{\nu, \max }$ (eq. [2]). There is a general scaling for the late-time R-band flux after the break $\left(t>t_{m}\right)$ $F_{\nu_{R}}(t) \propto \epsilon_{e}^{p-1} \epsilon_{B}^{(p-2) / 4} E_{52}^{(p+2) / 4} t^{-(3 p-2) / 4} \propto t_{m, R}^{3(p-1) / 4} \epsilon_{B}^{-1 / 4} E_{52}^{3 / 4} t^{-(3 p-2) / 4}$, where $t_{m, R}$ is the break time at the R-band. Sources with later breaks tend to be brighter. 


\section{MODELING GRB 021004}

The gamma-ray burst GRB 021004 was detected by the HETE II satellite (Shirasaki et al. 2002) and had an optical afterglow detected within minutes of the $\gamma$-ray burst (Fox et al. 2003b). The early light curves of the only other afterglows detected at such early times, GRBs 990123 (Akerlof et al. 1999) and 021211 (Fox et al. 2003a), have similar shapes, both showing a rapid initial decline followed by a slower decay, although GRB 021211 is fainter by about 3 magnitudes at similar epochs. In both sources, the initial rapidly decaying emission is interpreted as coming from the reverse shock of GRB ejecta running into a constant density medium (Sari \& Piran 1999; Li et al. 2003; Fox et al. 2003a; Wei 2003). The early optical afterglow of GRB 021004 shows a different behavior: it has a very slow initial decay of $t^{-0.4 \pm 0.1}$, followed by a steepening around day 0.1 into approximately $t^{-1}$ (Fox et al. 2003b). Kobayashi \& Zhang (2003) interpreted the early afterglow data in terms of emission from a combination of reverse and forward shocks expanding into a constant-density medium. Fox et al. (2003b) questioned this interpretation, and suggested instead a continued energy injection into the blast wave after the $\gamma$-ray burst to explain the initial slow decay. We propose that the slow decay is a natural consequence of the fast cooling $\left(\nu_{c}<\nu_{m}\right)$ expected in a wind model at early times and that the steepening is caused by the typical frequency $\nu_{m}$ passing through the optical band from above while still in the fast cooling regime. We show that this interpretation, besides fitting the R-band light curve, is in a reasonable agreement with the radio data available at several frequencies.

The free parameters that appear in the wind model can be estimated analytically using the formulae given in the previous section. Our identification of the break time $t_{m, R}=0.1$ day in the R-band light curve yields, using eq. (1), the relation $\epsilon_{e,-1}^{4 / 3} \epsilon_{B,-1}^{1 / 3} E_{52}^{1 / 3}=1.7$, for a redshift of $z=2.32$ (Chornock \& Filippenko 2002). For a cosmological model with $H_{0}=71 \mathrm{~km} \mathrm{~s}^{-1} \mathrm{Mpc}^{-1}, \Omega_{M}=0.27$, and $\Omega_{\Lambda}=0.73$, this redshift 
corresponds to $d_{L 1}=1.89$. At the break, the R-band flux is approximately $F_{\nu_{m}}=0.83 \mathrm{mJy}$, which yields a second relation $\epsilon_{e,-1}^{-1} \epsilon_{B,-1}^{-1 / 2} E_{52}^{1 / 2}=1.9$ from eq. (3). The wind density $A_{*}$ does not enter into either of the two relations, which enables us to express $\epsilon_{e}$ and $E_{52}$ in terms of $\epsilon_{B}: \epsilon_{e}=0.11 \epsilon_{B,-1}^{-1 / 3}$ and $E_{52}=4.0 \epsilon_{B,-1}^{1 / 3}$.

To constrain the wind density $A_{*}$, we note that the typical frequency $\nu_{m}$ decreases with time as $t^{-3 / 2}$. It should cross the $8.46 \mathrm{GHz}$ wavelength around day 140 . This is much later than the time $t_{0}$ for transition to slow cooling, which occurs around $t_{0}=3.7 \epsilon_{B,-1}^{2 / 3} A_{*}$ days, for typical parameters. Therefore, we can use eq. (2) to find the expected peak flux at 8.46 GHz. The result is

$$
F_{\nu_{m}, 8.46 \mathrm{GHz}}=0.59 \epsilon_{B,-1}^{2 / 3} A_{*} \mathrm{mJy}
$$

which for standard parameters is comparable to the R-band flux at the break $t_{m, R}$. The 8.46 GHz flux is observed at $598 \pm 33 \mu \mathrm{Jy}$ on day 5.7. If this flux is comparable to the peak flux at the time $t_{m}$ (which is true if the observed frequency is close to or beyond the self-absorption frequency; see CL00), then one can use eq. (4) to provide a rough estimate for $A_{*}$ in terms of $\epsilon_{B}: A_{*} \approx 1.0 \epsilon_{B,-1}^{-2 / 3}$.

The above analytic estimates assumed $p=2.5$, and are rather crude. They do indicate that the optical and radio data may be fitted with a wind model with parameters not far from the standard values. We now demonstrate that this is indeed the case using a numerical model. The model treats synchrotron emission from a spherical (trans-)relativistic blast wave propagating in an $r^{-2}$ density medium, with the light travel time effects, synchrotron self absorption and cooling included. It was previously applied to GRB 980508 (CL00), among others. Like GRB 980508, the decay of the R-band flux on the time scale of days and longer is relatively slow, with $F_{\nu} \propto t^{-1}$ approximately; the wiggles on the light curve of this source makes a precise determination of the decay slope difficult. The slope implies that $p$ is close to 2 , although the exact value is somewhat uncertain. We pick $p=2.1$, which 
corresponds to a decay slope of $\alpha=-(3 p-2) / 4=1.075$ in the optical.

After some experimentation, we find a solution that fits the R-band and radio data reasonably well with the following set of parameters: $\epsilon_{e}=0.1, \epsilon_{B}=0.1, E_{52}=10$, and $A_{*}=0.6$, which corresponds to a wind mass loss rate of $6 \times 10^{-6} \mathrm{M}_{\odot} \mathrm{yr}^{-1}$ (assuming a nominal wind speed of $10^{3} \mathrm{~km} \mathrm{~s}^{-1}$ ). Now all the parameters are approximately determined (within a factor $\sim 2$ ) because of the inclusion of self-absorption. The fits are shown in panels (a) and (b) of Fig. 1. We did not attempt to fit the bumps on the R-band light curve; they have been interpreted as arising from either late energy injections or inhomogeneities in the ambient density (Lazzati et al. 2002; Nakar, Piran \& Granot 2003; Heyl \& Perna 2003). The bumps introduce some uncertainty to the model parameters we obtained. Radio emission was detected at 4.86, 8.46, 15, 22.5 and $86 \mathrm{GHz}$ at various times (Frail \& Berger 2002; Berger, Frail \& Kulkarni 2002; Pooley 2002b,c; Bermer \& Castro-Tirado 2002). One upper limit each exists at 15, 232 and 347 GHz (Pooley 2002a; Bermer \& Castro-Tirado 2002; Wouterloot et al. 2002). The flux measurement of $2.5 \pm 0.3 \mathrm{mJy}$ at $86 \mathrm{GHz}$ flux at an average time of 1.5 days is particularly interesting. This flux is three times higher than the R-band flux at the break around day 0.1. It presents a problem to the identification of the break as $t_{m}$ in a constant density medium when the cooling frequency has already passed the R-band from below (Kobayashi \& Zhang 2002). In such a case, the maximum fluxes at lower frequencies should be the same as that of the R-band at the break, namely about $0.83 \mathrm{mJy}$, which is well below the $86 \mathrm{GHz}$ measurement. This discrepancy was also noted by Pandey et al. (2002).

The relatively high $86 \mathrm{GHz}$ flux is not a problem for our model, where the transition to slow cooling occurs around day 2, much later than day 0.1. In a wind model, the flux can be much higher in the radio (broadly defined to include millimeter and sub-millimeter wavelengths), particularly at early times when the cooling frequency is expected to be in 
the spectral region. This behavior shows up clearly in panel (b) of Fig. 1, where the peak fluxes at the three highest frequencies are all above $3 \mathrm{mJy}$. Such high fluxes are naturally expected in a wind model but not in an ISM model, as emphasized by Panaitescu \& Kumar (2000). However, it is difficult to make a strong case for wind interaction based on a single data point at $86 \mathrm{GHz}$. A stronger case can be made if the $8.46 \mathrm{GHz}$ flux starts to decline around 100 days, when the typical frequency $\nu_{m}$ is expected to pass through the frequency from above. This expectation needs to be modified in the case of an early jet break.

X-ray afterglows are observed with Chandra at two epochs. The first epoch started about 0.87 days after the burst and lasted for 88.1 ksec (Sako \& Harrison 2002a; Fox et al. 2003b). Within this epoch, the X-ray afterglow has a power-law spectrum, with index $\beta_{X}=-1.1 \pm 0.1$, and decays roughly as a power-law, with index $\alpha_{X}=-1.0 \pm 0.2$. Both are consistent with our model, where the cooling frequency $\nu_{c}$ around day 1 is well below the X-ray band, and $\beta_{X}$ and $\alpha_{X}$ are predicted to be -1.05 and -1.075 , respectively, for $p=2.1$. The fact that the temporal decay slope in the R-band, $\alpha_{O}$, is close to -1 indicates that the cooling frequency is below the R-band as well around this time, which is in agreement with the optical spectral index of $\beta_{O}=-1.07 \pm 0.06$ determined by Pandey et al. (2002) and $\beta_{O}=-0.96 \pm 0.03$ by Matheson et al. (2003) in the absence of a substantial host galaxy extinction (see also Bersier et al. 2003; Holland et al. 2002). Our best fit model yields an X-ray flux of $2.6 \times 10^{-13} \mathrm{erg} \mathrm{cm}^{-2} \mathrm{~s}^{-1}$ between 2 and $10 \mathrm{keV}$ at day 1.4 , close to the middle of the first observing epoch. It is lower than, but within a factor of two of, the mean flux of the entire epoch $4.3 \times 10^{-13} \mathrm{erg} \mathrm{cm}^{-2} \mathrm{~s}^{-1}$ (Sako \& Harrison 2002a). We therefore conclude that the wind model is consistent with the first epoch of X-ray observations.

The second epoch of X-ray observations started 52.23 days after the burst and yielded a 2-10 keV flux of $7.2 \pm 2.5 \times 10^{-16} \mathrm{erg} \mathrm{cm}^{-2} \mathrm{~s}^{-1}$ (Sako \& Harrison 2002b). The flux implies a decay slope between the two epochs of approximately $\alpha_{X}=-1.7$, which is steeper than that 
predicted in our spherical model. The steepening may be due to a jet break between the two epochs of observation, which was suggested to have occurred around day 7 by Pandey et al. (2002) and Holland et al. (2002) based on their interpretation of the (wiggly) R-band light curve. The jet break, if exists, should show up in a well-sampled radio light curve as well. Alternatively, the steepening could be due to a steepening of the energy distribution of nonthermal electrons well above the minimum energy of the electrons accelerated at the shock front (e.g., Li \& Chevalier 2001; Panaitescu \& Kumar 2002).

The question of a jet break is related to the energy in the source. The energy we find in a spherical model for the afterglow, $E_{52}=10$, is comparable to the isotropic burst energy in $\gamma$-rays, $5 \times 10^{52} \mathrm{ergs}$ (Bloom, Frail, \& Kulkarni 2003). Pandey et al. (2002) find, in a fit to the optical data through day 21 , that there is a break in the light curve at $t_{b}=7.6 \pm 0.3$ days. Bloom et al. (2003) interpret this as a jet break; the correction for collimated flow reduces the $\gamma$-ray energy by a factor of $\sim 40$. However, Fig. 1 shows that the variability in the light curve and the late light curve points make a clear designation of the jet break difficult. In a wind model, the jet break evolves slowly (Kumar \& Panaitescu 2000), which makes any determination of a jet break from afterglow data more uncertain.

The mass loss rate we deduced is typical for a Wolf-Rayet type wind. There are other indications that GRB 021004 may be interacting with a Wolf-Rayet type wind. Wolf-Rayet winds are thought to be clumpy (e.g., Hamann \& Koesterke 1998), and the clumpiness may provide an explanation for the prominent bumps on the R-band light curve of GRB 021004 (Lazzati et al. 2002; Nakar et al. 2002; Heyl \& Perna 2003). In addition, there are multiple absorption components in the spectrum of the afterglow, separated by speeds up to $3000 \mathrm{~km} \mathrm{~s}^{-1}$. These components could come from substructures in a Wolf-Rayet wind (Mirabal et al. 2002; Schaefer et al. 2002). Interestingly, GRB 990510, which is best modeled by interaction with a constant density medium (CL00; Panaitescu \& Kumar 2002), 
shows a smoothly evolving optical afterglow (Stanek et al. 1999).

GRB 021004 differs from the other two GRBs with detected early afterglows (GRBs 990123 and 021211) in several ways: it has a slow decay in the R-band light curve followed by a steepening rather than a steep decline followed by a flattening (which occurs at a much earlier time than the break in GRB 021004), a higher optical flux at late times after the break, and a bright, long-lived radio afterglow. The early emission from GRB 990123 was convincingly interpreted as coming from the reverse shock of a blast wave expanding into an ISM (Sari \& Piran 1999; Mészáros \& Rees 1999), and the late-time afterglow data are consistent with an ISM model (e.g., Panaitescu \& Kumar 2002). The afterglow of GRB 021211 resembles that of GRB 990123, and was interpreted similarly (Fox et al. 2003a; Li et al. 2003; Wei 2003). The rate of initial decline in the R-band flux of approximately $t^{-2}$ (GRB 990123) or shallower (GRB 021211) is difficult to reproduce in the reverse shock of a wind model. The available data on the three early afterglows therefore appear to point to two types of GRB ambient environments: ISM and a stellar wind. These two types of environments are also inferred in the detailed modeling of a larger number of afterglows at later times (CL00; Panaitescu \& Kumar 2002; Price et al. 2002).

The evidence for wind and constant density environments in the early afterglow evolution supports the hypothesis that these represent different progenitors (Chevalier \& Li 1999; CL00) because a small radial distance from the star is explored $\left(\lesssim 10^{17} \mathrm{~cm}\right)$ where a freely expanding wind is expected. A prediction of the CL99 scenario is that wind interaction should be correlated with supernova light; this hypothesis was supported by GRB 011121 (Price et al. 2002). However, GRB 021211, which has an early afterglow indicating ISM interaction, shows some evidence for a supernova-like bump in the light curve (Fruchter et al. 2002; Testa et al. 2003). GRB 020405 is another case of apparent ISM interaction and a supernova-like bump (Berger et al. 2003). Spectra confirming the 
supernova nature of light curve bumps, as well as detailed afterglow observations, are needed to clarify the situation. Long term monitoring of radio afterglows will be crucial in testing the wind model of early afterglows (by examining the evolution of $\nu_{m}$ ), and in determining the wind density. Another prediction of the wind model is that the early optical emission before the break $\left(t<t_{m}\right)$ should have the spectrum $F_{\nu} \propto \nu^{-1 / 2}$, which is a flatter spectrum than is typically observed in optical afterglows. In addition, the break is chromatic, occurring at a later time for a longer wavelength, which can be tested with densely sampled IR observations.

Support for this work was provided in part by NASA. 


\section{REFERENCES}

Akerlof, C. W., et al. 1999, Nature, 398, 400

Berger, E., Frail, D. A., \& Kulkarni, S. R. 2002, GCN 1613

Berger, E., Soderberg, A. M., Frail, D. A., \& Kulkarni, S. R. 2003, ApJ, submitted (astro-ph/0301634)

Bersier, D., et al. 2003, ApJ, 584, L43

Bloom, J. S., Frail, D. A., \& Kulkarni, S. R. 2003, ApJ, submitted (astro-ph/0302210)

Bremer, M., \& Castro-Tirado, A. J. 2002, GCN 1590

Chevalier, R. A., \& Li, Z.-Y. 1999, ApJ, 520, L29 (CL99)

Chevalier, R. A., \& Li, Z.-Y. 2000, ApJ, 536, 195 (CL00)

Chornock, R., \& Filippenko, A. V. 2002, GCN 1605

Fatkhullin, T. A., Komarova, V. N., Moiseev, A. V. 2002, GCN 1717

Fox, D. W., et al. 2003a, ApJ, 586, L5

Fox, D. W., et al. 2003b, Nature, 422, 284

Frail, D. A., \& Berger, E. 2002, GCN 1574

Frail, D. A., Waxman, E., \& Kulkarni, S. R. 2000, ApJ, 537, 191

Fruchter, A., et al. 2002, GCN 1781

Granot, J., \& Sari, R. 2002, ApJ, 568, 820

Hamann, W.-R., \& Koesterke, L. 1998, A\&A, 335, 1003 
Heyl, J. S., \& Perna, R. 2003, ApJ, 586, L13

Holland, S. T., et al. 2002, AJ, submitted (astro-ph/0211094)

Kobayashi, S., \& Zhang, B. 2003, ApJ, 582, L75

Kumar, P., \& Panaitescu A. 2000, ApJ, 541, L9

Kumar, P., \& Panaitescu, A. 2000, ApJ, 541, L51

Lazzati, D., Rossi, E., Covino, S., Ghisellini, G., \& Malesani, D. 2002, A\&A, 396, L5

Li, W., Filippenko, A. V., Chornock, R., \& Jha, S. 2003, ApJ, 586, L9

Li, Z.-Y., \& Chevalier, R. A. 2001, ApJ, 551, 940

Matheson, T., et al. 2003, ApJ, 582, L5

Matsumoto, K., et al. 2002, GCN 1594

Mészáros, P. \& Rees, M. J. 1997, ApJ, 476, 232

Mészáros, P. \& Rees, M. J. 1999, MNRAS, 306, L39

Mirabal, N., Halpern, J. P., Chornock, R., \& Filippenko, A. V. 2002, GCN 1618

Nakar, E., Piran, T., \& Granot, J. 2002, New Astr., submitted (astro-ph/0210631)

Panaitescu, A., \& Kumar, P. 2000, ApJ, 543, 66

Panaitescu, A., \& Kumar, P. 2002, ApJ, 571, 779

Pandey, S. B., et al. 2002, BASI, submitted (astro-ph/0211108)

Pooley, G. 2002a, GCN 1575

Pooley, G. 2002b, GCN 1588 
Pooley, G. 2002c, GCN 1604

Price, P. A., et al. 2002, ApJ, 572, L51

Rhoads, J. E. 1997, ApJ, 487, L1

Sako, M., \& Harrison, F. A. 2002a, GCN 1624

Sako, M., \& Harrison, F. A. 2002b, GCN 1716

Sari, R. \& Piran, T. 1999, ApJ, 517, L109

Sari, R., Piran, T., \& Halpern, J. P. 1999, ApJ, 519, L17

Sari, R., Piran, T., \& Narayan, R. 1998, ApJ, 497, L17

Schaefer, B. E., et al. 2002, ApJ, submitted (astro-ph/0211189)

Shirasake, Y., et al. 2002, GCN 1565

Stanek, K. Z., Garnavich, P. M., Kaluzny, et al. 1999, ApJ, 522, L39

Testa, V., et al. 2003, GCN 1821

Wei, D. M. 2003, preprint (astro-ph/0301345)

Weidinger, M., et al. 2002, GCN 1573

Wouterloot, J., et al. 2002, GCN 1627 


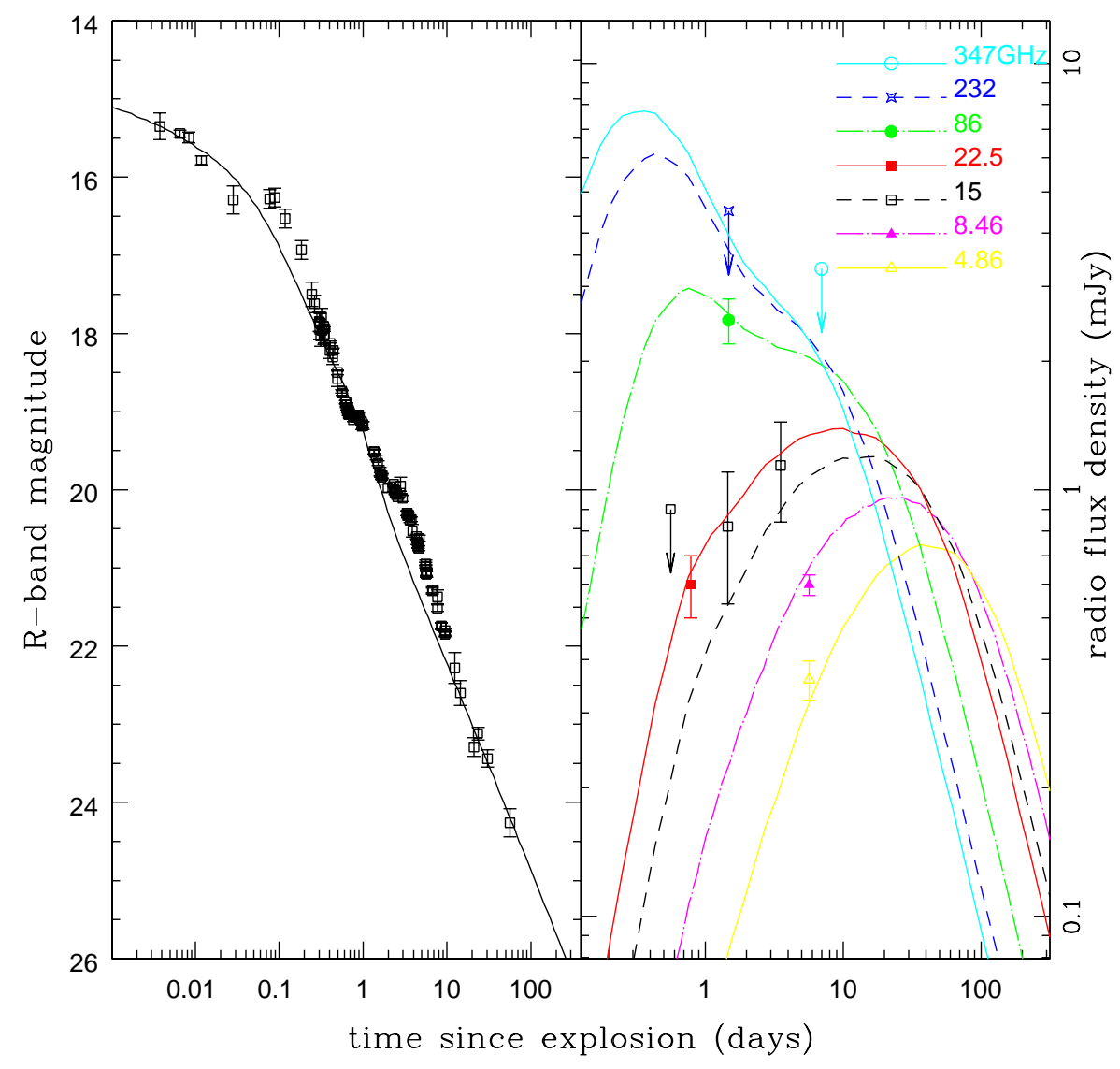

Fig. 1. - Wind interaction model for the afterglow of GRB 021004. The optical data are taken from the papers Fox et al. (2003b), Bersier et al. (2003), and Holland et al. (2003) and the GCN notices Matsumoto et al. (2002), Weidinger et al. (2002), Mirabal et al. (2002) and Fatkhullin et al. (2002), with the modest amount of Galactic extinction corrected. The radio data are taken from Frail \& Berger (2002; 22.5 GHz), Berger, Frail \& Kulkarni (2002; 4.86 and $8.46 \mathrm{GHz}$ ), Pooley (2002b,c; $15 \mathrm{GHz}$ ), and Bermer \& Castro-Tirado (2002; 86 GHz). The upper limits at 15, 232 and 347 GHz are given in Pooley (2002a), Bermer \& Castro-Tirado (2002) and Wouterloot et al. (2002). The lines are the light curves from the wind model described in the text. 